\title{
CONDICIONES DE TRABAJO Y BIENESTAR/MALESTAR DOCENTE EN PROFESORES DE ENSEÑANZA MEDIA DE SANTIAGO DE CHILE*
}

\author{
Rodrigo Cornejo Chávez
}

\begin{abstract}
RESUMEN: Esta investigación busca analizar conjuntamente las condiciones de trabajo docente (materiales y psicosociales) y el bienestar/malestar de los profesores de enseñanza secundaria de Santiago, Chile. Su diseño es multivariado, descriptivo/transversal, correlacional y explicativo (siendo bienestar/malestar la variable dependiente para la construcción del modelo de regresión múltiple). La información fue recogida durante el año 2008, en una muestra representativa para los establecimientos educacionales con subvención estatal de Santiago. Se concluye que los docentes perciben condiciones de trabajo precarias y altos niveles de demanda laboral (intensificación del trabajo). Se construye un modelo explicativo del bienestar/malestar de los docentes, que consta de siete variables, de las cuales dos se destacan por su alto poder explicativo: la "significatividad en el trabajo" y las "demandas laborales".
\end{abstract}

Palabras clave: Condiciones de trabajo. Bienestar y salud docente. Malestar docente.

\section{SECONDARY SCHOOL TEACHERS' WORKING CONDITIONS and Well-being/Discomfort in Santiago, Chile}

ABSTRACT: This research analyzes teachers' material and psychosocial working conditions and well-being/discomfort in Chilean secondary school. It has a multivariate, descriptive/cross-sectional,

* Investigación financiada por el Fondo Nacional de Investigación y Desarrollo en Educación (FONIDE), del Ministerio de Educación de Chile.

** Académico del Departamentos de Psicología y Educación, investigador del Observatorio Chileno de Politicas Educativas (оресн), Universidad de Chile. E-mail: rocornejo@uchile.cl 
correlational and explanatory design-wellness/discomfort being the dependent variable in the construction of this multiple regression model. Information was collected along 2008 from a representative sample of educational establishments funded by the State of Santiago. It concludes that teachers perceive the precariousness of their working conditions and the high levels of labor demand (work intensification). An explanatory model of teachers' well-being/discomfort is constructed, which consists of seven variables, two of which are outlined for their high explanatory power: "meaningfulness in work" and "labor demands".

Key words: Working conditions. Teachers' well-being and health. Teachers' discomfort

\section{Planteamiento del problema}

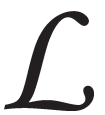

os estudios sobre trabajo, bienestar/malestar y salud de los docentes han crecido en cantidad y en contundencia teórica y metodológica durante los últimos veinte años (pese a seguir presentando retraso respecto de la investigación realizada en el sector del trabajo industrial). Dos grandes fenómenos, ocurridos en la historia reciente, han jaloneado este desarrollo. Por un lado, las profundas transformaciones que ha experimentado el trabajo docente durante las últimas décadas, de la mano de las mutaciones socioculturales del capitalismo post industrial y de la masificación de la escuela. Por otro, el significativo aumento de los problemas de salud mental y malestar en la profesión docente, referidos en la literatura especializada a nivel mundial (Tenti, 2006; Montgomery y Rupp, 2005; UNEsCO, 2005; Kyriacou, 2001; Valdivia et al., 2003; Hargreaves, 1997; Esteve et al., 1997; Cornejo, 2007).

Estos estudios han aportado abundante evidencia que respalda la existencia de intensas relaciones entre las condiciones laborales y el bienestar y la salud de los docentes, y han ayudado a superar una visión psicologicista que entendía los fenómenos del malestar y la enfermedad en el trabajo como un asunto individual. También han demostrado que no son los oficios, en sí mismos, los que generan enfermedad, sino las condiciones concretas y específicas en que se realiza el trabajo, lo que se ha denominado factores de riesgo (Parra, 2007, 2001; van der Doef y Maes, 1999a; Mendel, 1993; Dejours, 1990). 
En los últimos años, una de las líneas de investigación más promisorias en este campo ha sido la búsqueda de modelos explicativos del bienestar/malestar docente basados en aspectos psicosociales del trabajo. Los modelos que cuentan con mayor sustento empírico en el sector docente son el de "desajuste esfuerzo-recompensa" y el modelo "control-demanda-apoyo social" (Parra, 2001; Karasek y Theorell, 1990; Verhoeven et al., 2003). Estos modelos enfatizan en la importancia de aspectos como "nivel de demanda laboral percibida", "control y autonomía decisional", "apoyo social", "valoración social” y "significatividad" del trabajo. Desde el punto de vista teórico, estos modelos tienen puntos de coincidencia con aportes clásicos de la sociología y psicopatología del trabajo, que plantean relaciones entre el grado de poder que tiene el trabajador sobre su actividad y los niveles de satisfacción laboral, productividad y bienestar/malestar psicológico (Mendel, 1993; Dejours, 1990).

Por otra parte, los indicadores de salud laboral docente más utilizados en los estudios cuantitativos actuales pueden ubicarse en tres grandes campos: salud física (trastornos músculo esqueléticos, disfonías etc.), salud mental (trastornos depresivos y ansiosos), malestar o bienestar psicológico (agotamiento emocional, burnout, satisfacción laboral, compromiso). El mayor programa de investigación en Europa, actualmente en curso, sobre trabajo y salud en profesores, llamado EUROTEACH, utiliza el constructo de "bienestar/salud" (Verhoeven et al., 2003; Rasku y Kinnunen, 2003). En este estudio utilizamos el constructo de bienestar/malestar docente y evaluamos distintos indicadores de condiciones materiales y psicosociales de trabajo, basados en los modelos antes descritos.

\section{Objetivos}

El objetivo general de la investigación es analizar el bienestar/ malestar laboral en docentes de educación secundaria de Santiago, y la asociación e influencia que tienen sobre éste las condiciones materiales y psicosociales del trabajo docente.

Los objetivos específicos de esta investigación pretenden responder a las siguientes preguntas guías: ¿Cómo son las condiciones materiales y psicosociales de trabajo de los profesores de Santiago? ¿Cuál es 
el perfil de bienestar/malestar docente de las profesoras y profesores de educación secundaria de Santiago? ¿Cuál es la relación existente entre el bienestar/malestar de los docentes, sus condiciones de trabajo y algunos aspectos personales de los docentes?

\section{Metodología}

Esta investigación es de carácter cuantitativo, multivariado y transversal. El nivel de conocimientos que se desea alcanzar es de tipo correlacional. El estudio es representativo de la ciudad de Santiago de Chile. La población objetivo del estudio corresponde a docentes que imparten clases a estudiantes de enseñanza secundaria de colegios municipales (con administración municipal y financiamiento estatal) y particulares subvencionados (con financiamiento estatal y administración privada) de las comunas urbanas de Santiago.

Se realizó un muestreo probabilístico uni etápico, aleatorio, estratificado de manera independiente según la modalidad de enseñanza (científica/humanista o técnica/profesional) y dependencia administrativa del establecimiento educacional (municipal o particular con subvención estatal), sin afijación proporcional y utilizando los colegios como conglomerados de docentes. Se realizó una selección aleatoria de establecimientos educacionales a través de Salto Sistemático ordenado por comuna del colegio para asegurar representatividad en esta condición. Así se seleccionaron 45 establecimientos educacionales a los que se les aplicó una encuesta de autorreporte a sus profesores. Si bien el diseño muestral asume a los establecimientos como conglomerados, no fue posible encuestar a todos los docentes de los colegios muestreados a causa de ausencias y no respuestas. Pese a ello, el porcentaje de docentes que respondió la encuesta en cada establecimiento fue aproximadamente de un $83 \%$, con lo cual se cumple con los estándares requeridos para este tipo de estudios.

\section{Instrumentos de recolección de información} tario:

Se utilizó un instrumento central y un instrumento complemen-

1. Cuestionario de autorreporte: este es un cuestionario estructurado con preguntas cerradas que se construyó especialmente para 
este estudio, utilizando diversas subescalas de instrumentos de reconocida validez y confiabilidad para medir las variables y conceptos estudiados. ${ }^{1}$ El cuestionario creado fue aplicado previamente en una muestra piloto para evaluar su comprensión, consistencia y fiabilidad.

2. Lista de chequeo sobre condiciones materiales de trabajo: la lista de chequeo se centra en aspectos de infraestructura y fue construida en base al instrumento utilizado por UNESCO en su estudio sobre Salud Laboral y Condiciones de Trabajo en América Latina (2005). Fue aplicada por observadores externos, previamente entrenados, en una sub muestra de veinte establecimientos.

\section{Variables estudiadas}

1. Variables de identificación del docente y del establecimiento.

2. Indicadores de bienestar/malestar docente: se recogió información acerca de agotamiento emocional, distancia emocional, sensación de falta de logro (estos tres indicadores constituyen el modelo trifactorial de burnout), síntomas generales de ansiedad y depresión, satisfacción vocacional e índice de enfermedades presuntas. A partir de estas variables se construyó un índice general de bienestar docente.

3. Condiciones materiales de trabajo: se recogió información acerca de un conjunto de indicadores que hemos agrupado en torno a cuatro ejes: empleo y precariedad (remuneraciones, estabilidad laboral, tener además otro trabajo, sindicalización), jornada laboral (relación entre horas trabajadas y horas contratadas; porcentaje de horas lectivas en la jornada laboral, descanso), exigencias ergonómicas (postura incómoda, forzar la voz, ruido, iluminación, temperatura), materiales educativos (disponibilidad de materiales de trabajo educativo y pertinencia del material de trabajo educativo) e infraestructura (a través de la lista de chequeo por observación externa).

4. Condiciones psicosociales de trabajo: las condiciones psicosociales del trabajo están compuestas por siete variables: demandas 
del trabajo, control en el trabajo, apoyo social, significatividad, entorno social del trabajo, cantidad de alumnos a cargo y existencia o no de selección de estudiantes.

5. Variables personales: la conforman dos variables: estrategias individuales de afrontamiento de situaciones estresantes y autoestima.

\section{Procesamiento y análisis de la información}

Con los datos obtenidos del cuestionario de autorreporte se realiza estadística descriptiva e inferencial (test de diferencias significativas) respecto de las condiciones de bienestar/malestar, y de las condiciones materiales y psicosociales de trabajo de los docentes, diferenciando según dependencia administrativa del colegio y otras características relevantes del docente y del colegio.

Se aplicaron técnicas de reducción de datos, como análisis factorial confirmatorio, para construir los constructos bienestar/malestar laboral, condiciones materiales y psicosociales de trabajo. Además, se construyó un modelo explicativo de regresión lineal múltiple, para estimar el nivel de bienestar/malestar laboral de los docentes (variable dependiente) en función de las condiciones materiales y psicosociales de trabajo y variables personales de los docentes (variables independientes). La información obtenida a partir de la lista de chequeo se analiza con estadística descriptiva.

Principales resultados de la investigación

\section{Descripción del bienestar/malestar docente}

Para analizar el nivel de bienestar de los docentes se creó un Índice General de Bienestar (IGB), a partir de los siguientes indicadores ponderados: agotamiento emocional, distancia emocional, sensación de falta de logro, síntomas generales de ansiedad/depresión, indicador de enfermedades presuntivas (reportadas por los propios docentes), satisfacción vocacional. Los resultados obtenidos en este índice mostraron diferencias significativas por modalidad de enseñanza. Los docentes que 
trabajan en liceos científico-humanistas presentan niveles significativamente más bajos de bienestar, en comparación con aquellos que trabajan en liceos técnico-profesionales.

En relación a los indicadores relacionados con el burnout, destacamos los siguientes resultados: alrededor de la cuarta parte de los docentes de educación secundaria de Santiago presenta niveles altos de agotamiento emocional $(26,6 \%)$, distancia emocional $(20,1 \%)$ y sensación de falta de logro $(28,6 \%)$. El agotamiento emocional, se presentó en niveles significativamente mayores en las profesoras mujeres $(30,3 \%)$ en comparación con los docentes varones $(22,3 \%)$. En cambio, se observó que los hombres presentan una tendencia significativa a tener mayores niveles de distancia emocional $(23,9 \%)$ en comparación con las docentes mujeres (16,9\%). En tanto, los resultados obtenidos en la escala de falta de logro no reportan diferencias significativas por género. Los tres indicadores mencionados constituyen el modelo trifactorial de burnout de Maslach, el cual se confirma para la población estudiada, con un peso factorial preponderante del indicador de agotamiento emocional (30\%).

En cuanto a la sintomatología ansiosa y depresiva, solo un $6 \%$ de los docentes de la muestra presentan niveles altos al ser aplicado el screening Test GHQ-12 de Goldberg.

Por otra parte, los profesores y profesoras reportaron haber tenido las siguientes enfermedades durante los últimos dos años: bronquitis $(32,1 \%)$; colon irritable $(21,6 \%)$, disfonía $(18,6 \%)$, tendinitis $(16,6 \%)$, estrés $(13,9 \%)$, depresión $(13,2 \%)$, hipertensión $(11,5 \%)$. Se observaron diferencias significativas por sexo y tramo erario. Las mujeres tienden a enfermarse más que los hombres, en tanto, a mayor edad, se presentan mayores niveles de enfermedad.

En términos del indicador de bienestar psicológico "satisfacción vocacional”, se observaron diferencias significativas por dependencia. Los docentes que trabajan en liceos municipales presentan mayores niveles de satisfacción vocacional que quienes trabajan en liceos particulares. Si bien no existen puntos de corte para esta escala, se puede afirmar que los docentes estudiados presentan niveles aceptables de satisfacción vocacional, es decir, de compromiso con el contenido de la profesión, especialmente en el sector municipal. 


\section{Descripción de condiciones materiales de trabajo}

Empleo y precariedad: el empleo reportado por los profesores y profesoras en la ciudad de Santiago tiende a la precariedad. En su mayoría, los docentes reciben una remuneración baja en consideración al nivel profesional de la carrera, el $75 \%$ de ellos no se encuentran sindicalizados, se mantienen apenas tres años en un mismo establecimiento educacional como promedio, y los más relevante, cerca del $50 \%$ necesita (u opta por tener) otro empleo. Estos indicadores son significativamente más positivos en los trabadores del sector municipal.

Jornada laboral: los docentes de educación media reportan trabajar aproximadamente 10 horas extras no remuneradas a la semana (lo que representa casi un tercio de su contrato laboral). Además reportan falta de instancias de descanso y desconexión durante la jornada laboral. Es en los colegios científico-humanistas donde esta sobre-exigencia se da en mayor medida. En relación a las horas lectivas que reportan los docentes, el presente estudio muestra que los profesores de enseñanza media de Santiago están dedicando más de un $85 \%$ de su jornada laboral al trabajo en aula, lo que superan el máximo legal permitido de $75 \%$. Estos preocupantes resultados son homogéneos para los docentes, independientemente de las características de cada colegio.

Pertinencia y disponibilidad de materiales pedagógicos: los materiales pedagógicos de apoyo a la docencia son percibidos por la mayoría de los docentes como insuficientes $(66 \%)$ y poco pertinentes (51\%). Situación que se acentúa en colegios técnico-profesionales.

Escala de exigencias ergonómicas: las exigencias ergonómicas son percibidas como excesivas por un porcentaje mayoritario de los docentes, especialmente en lo que respecta a tener que estar de pie durante la jornada laboral (86\%), tener que forzar la voz (76\%) y estar expuesto a cambios bruscos de temperatura (49\%). Estas exigencias son percibidas en un nivel significativamente mayor, por los docentes de establecimientos municipales.

Condiciones de la infraestructura: estas condiciones fueron observadas con la lista de chequeo en 20 colegios. Llama la atención que aún existan colegios que no cuenten con sala de profesores; como 
también es lamentable que en casi la mitad de los colegios no se cuente con un mobiliario en buen estado para este lugar, que es dónde los docentes deben realizar parte importante de sus tareas, además de ser un espacio de reunión y descanso. Cuando se revisa el estado de las salas de clase, nos encontramos con falta de mobiliario adecuado para el profesor, y mala iluminación. A lo anterior se agrega que los docentes deben realizar sus clases en salas que no cuentan con el aislamiento para los ruidos externos, lo cual implica necesariamente que deben forzar su voz para ser escuchados por todo el alumnado.

\section{Descripción y análisis de las condiciones psicosociales del trabajo docente}

Demanda laboral: se consultó a los docentes sobre: ambigüedad de rol (tareas poco claras o contradictorias entre si), presión excesiva de trabajo y falta de tiempo para realizar éste, e interacción problemática con estudiantes. Los resultados indican que son los profesores de los establecimientos municipalizados quienes perciben significativamente mayores niveles de demandas laborales, en comparación con docentes del sector particular subvencionado. Igual es el caso de los docentes de colegios técnico-profesionales, quienes perciben significativamente mayores niveles de demandas laborales que el resto de sus colegas. Sin embargo, se observó un nivel de variabilidad de los datos relativamente baja, por lo que se puede afirmar que la percepción de excesiva carga de trabajo es más bien homogénea entre los docentes.

Control sobre el trabajo: se evaluaron aspectos relacionados con la toma de decisiones laborales y la oportunidad que tienen los docentes para continuar su formación. En este indicador se observan resultados relativamente altos en relación a las otras escalas psicosociales, es decir, en general los profesores perciben contar con altos rangos de toma de decisión sobre su trabajo. Se aprecian diferencias por modalidad, siendo los docentes de establecimientos técnico-profesionales quienes perciben mayor control sobre su trabajo.

Apoyo social: se consultó a los docentes acerca del apoyo que reciben de sus directivos, jefes de unidad técnico-pedagógica y compañeros de trabajo. Los docentes sienten que el apoyo social que les entrega la dirección de los establecimientos es significativamente más 
bajo que el que reciben de parte de la unidad técnico-pedagógica del liceo y de parte de sus propios colegas. Es en los establecimientos particulares subvencionados donde los docentes perciben tener un mayor apoyo social en comparación con los otros establecimientos.

Significatividad en el trabajo: se entiende como la posibilidad de vincular el trabajo con valores u objetivos que no son de carácter instrumental (como sueldo y trabajo), de manera de otorgarle un sentido trascendente. Los profesores y profesoras tienden a mostrar un nivel de significatividad en el trabajo similar y cercano a la media, aún cuando trabajen en diferentes tipos de colegios.

Condiciones institucionales para las prácticas reflexivas: los docentes sienten que existe un número adecuado de reuniones colectivas de trabajo, pero solo un $25 \%$ de ellos creen que éstas permiten el desarrollo de reflexiones colectivas sobre el contenido pedagógico y el sentido del trabajo.

Entorno social del trabajo: se consultó a los docentes respecto del nivel de violencia y seguridad percibido. Los profesores de los colegios particulares subvencionados $(65,4 \%)$ encuentran de forma significativa el entorno de su escuela más seguro en comparación con los colegios municipales $(49,7 \%)$. Casi un tercio de los docentes del sector municipal afirma haber sido amenazado o afectado por actos de violencia en la escuela, cifra que es significativamente mayor a la mostrada por los particulares subvencionados, en donde sólo un $10 \%$ concuerdan con esta afirmación.

Alumnos a cargo: en general, los docentes tienen a cargo un promedio de 8 cursos a los que le hacen clases, en tanto, se observó un promedio de un curso como jefatura para cada docente. En cuanto al número de alumnos promedio por sala de clases, cerca de un $40 \%$ de los docentes deben atender a más de 39 alumnos por sala, un 54\% trabajan con salas de entre 30 y 39 estudiantes y sólo un $6 \%$ lo hacen en aulas con menos de 30 estudiantes. Al respecto no se apreciaron diferencias significativas por dependencia o modalidad educativa del establecimiento.

Selección de estudiantes: se trata de un asunto altamente relevante en el contexto educativo chileno, en el cual se permite que los colegios realicen procesos de selección de estudiantes. Más del 70\% de los profesores del sector municipal afirman que no existe selección, cifra 
significativamente mayor al 58\% que opinan de la misma forma en los colegios particulares subvencionados.

\section{Relaciones entre condiciones de trabajo y bienestar/malestar docente}

Todos los indicadores de condiciones psicosociales del trabajo docente muestran correlaciones, leves o moderadas, con el IGB y con los indicadores específicos de bienestar/malestar. En el caso de los indicadores de condiciones materiales de trabajo, sólo algunos de ellos muestran correlaciones débiles con el IGB y con los indicadores de bienestar/malestar. Estos resultados eran esperables, pues las relaciones entre condiciones materiales y bienestar docente no son directas. Además algunos indicadores de condiciones materiales presentan niveles de variabilidad tan bajos, que hacen imposible una covariación con los indicadores de bienestar. Los indicadores de aspectos psicológicos personales de los docentes muestran correlaciones leves o no presentan correlaciones con el IGB y los indicadores de bienestar/malestar. Estas relaciones más débiles eran esperables, pues como señala la literatura de salud laboral, las relaciones entre los elementos de contexto y las características personales de los docentes no son lineales y se encuentran actualmente en debate.

\section{Modelo explicativo de las relaciones entre bienestar docente y condiciones de trabajo}

Se realizó un análisis de regresión múltiple, teniendo como variable dependiente el IGB docente y como variables independientes explicativas aquellos indicadores de condiciones de trabajo y de aspectos psicológicos individuales que mostraron, a lo largo de los análisis anteriores, mayores niveles de relación con la variable dependiente y menores niveles de colinealidad entre sí, como una forma de evitar al máximo los errores de especificación (inclusión de variables irrelevantes y omisión de variables relevantes).

El modelo explicativo definitivo incorporó siete variables independientes y tuvo un buen ajuste llegando a explicar el 46,5\% de la varianza del bienestar/malestar de los docentes. Tres de las variables explicativas corresponden a aspectos psicosociales del trabajo (significatividad, demanda y selección), una variable corresponde a condiciones materiales 
Condiciones de trabajo y bienestar/malestar docente en profesores...

(exigencias ergonómicas), dos variables corresponden a condiciones estructurales de trabajo (dependencia municipal y vulnerabilidad de los estudiantes) y una variable corresponde a aspectos personales de los docentes (capacidad de alejarse y tomar distancia de las condiciones estresantes). En la siguiente tabla se puede apreciar un resumen de modelo encontrado:

\section{Tabla 1}

Resumen del modelo bienestar/malestar

\begin{tabular}{|c|c|c|c|c|}
\hline \multirow{2}{*}{ Model } & $\mathrm{R}$ & $\mathrm{R}$ Square & \multicolumn{2}{|c|}{} \\
\cline { 2 - 5 } & R Square Change & F Change & Adjusted R Square & Std. Error of the Estimate \\
\hline 1 &, $518(\mathrm{a})$ &, 269 &, 267 & 10,45343 \\
2 &, $627(\mathrm{~b})$ &, 394 &, 392 & 9,52640 \\
3 &, $640(\mathrm{c})$ &, 410 &, 407 & 9,40729 \\
4 &, $658(\mathrm{~d})$ &, 433 &, 429 & 9,23067 \\
5 &, $671(\mathrm{e})$ &, 450 &, 445 & 9,09871 \\
6 &, $682(\mathrm{f})$ &, 465 &, 459 & 8,98382 \\
7 &, $687(\mathrm{~g})$ &, 472 &, 465 & 8,93098 \\
\hline
\end{tabular}

(a) Predictores: (Constante), Indicador de Significatividad. (b) Predictores: (Constante), Indicador de Significatividad, Indicador de Demandas del Trabajo. (c) Predictores: (Constante), Indicador de Significatividad, Indicador de Demandas del Trabajo, MUNicipal. (d) Predictores: (Constante), Indicador de Significatividad, Indicador de Demandas del Trabajo, municipal, IVE. (e) Predictores: (Constante), Indicador de Significatividad, Indicador de Demandas del Trabajo, municipal, IVE, Indicador de Condiciones Ergonómicas. (f) Predictores: (Constante), Indicador de Significatividad, Indicador de Demandas del Trabajo, municipal, IVE, Indicador de Exigencias Ergonómicas, Capacidad de distanciarse de la situación estresante. (g) Predictores: (Constante), Indicador de Significatividad, Indicador de Demandas del Trabajo, municipal, ive, Indicador de Exigencias Ergonómicas, Capacidad de distanciarse de la situación estresante, Selección Variable Dependiente: Índice General de Bienestar/Malestar.

Como se observa en el resumen del modelo, el indicador de "significatividad en el trabajo" explica un 26,7\% de la variabilidad en el Îndice General de Bienestar (IGB), el indicador "demandas del trabajo" agrega un $12,5 \%$ a la explicación de la variabilidad del IGB, la condición de "dependencia municipal" del establecimiento del docente agrega un $1,5 \%$ a la explicación, el índice de vulnerabilidad educativa 
agrega un 2,2\% a esta explicación, el indicador de exigencias ergonómicas explica un 1,6\% de la variabilidad del IGB, la capacidad de distanciarse de la situaciones estresantes explica un 1,4\%, y la existencia de selección de alumnos en el establecimiento explica un 0,6\% de la variabilidad del IGB.

De acuerdo a este análisis, la significatividad en el trabajo y posteriormente el nivel de demandas en el trabajo percibidas por los docentes, son las condiciones de trabajo que explican un mayor porcentaje de la variabilidad del IGB docente. En otras palabras, el nivel de bienestar/malestar de los docentes de educación secundaria de Santiago varía en íntima relación con la capacidad de otorgar un sentido no instrumental al trabajo (un propósito o un objetivo moral) y con el nivel de carga de trabajo percibida (ambigüedad de rol, presión excesiva de trabajo y tiempo e interacción problemática con estudiantes).

\section{Conclusiones}

El análisis técnico del comportamiento estadístico de las escalas utilizadas, que incluyó adaptación de contenidos, pilotaje, análisis de fiabilidad y validez, permite poner a disposición de la comunidad educativa y científica un conjunto de instrumentos válidos y fiables para evaluar un conjunto de indicadores sobre condiciones de trabajo y bienestar/malestar docente. La totalidad de las escalas y subescalas teóricas utilizadas para evaluar bienestar/malestar docente y condiciones de trabajo presentan niveles de fiabilidad adecuados o buenos: agotamiento emocional (,911); distancia emocional (,697), falta de logro (,755), síntomas generales de ansiedad/depresión (,888), satisfacción vocacional $(, 87)$, ambigüedad de rol $(, 813)$, presión de tiempo $(, 822)$, interacción problemática con estudiantes $(, 903)$ autoridad para decidir (,709), oportunidades de formación (,902) apoyo social de la dirección $(, 854)$, apoyo social de la unidad técnico-pedagógica $(, 878)$, apoyo social de colegas $(, 821)$, significatividad del trabajo $(, 822)$, autoestima docente $(, 775)$. Los factores teóricos subyacentes a las diferentes escalas se confirmaron.

Respecto al nivel de bienestar laboral docente, podemos decir que alrededor de un tercio de los docentes de Santiago presentan niveles altos, en al menos una de las escalas burnout del modelo trifactorial 
de Maslach. Esto resulta preocupante, puesto que estas respuestas psicológicas pueden afectar seriamente la construcción de climas afectivos de aula adecuados y las expectativas de los docentes hacia sus estudiantes. Los porcentajes de enfermedad reportados por los docentes superan a la media nacional para adultos y son particularmente preocupantes, en la medida que afectan la salud general o las herramientas corporales del trabajo docente (las manos y la voz). Por otra parte, el que solo un 6\% de los docentes de educación secundaria de Santiago presenten niveles altos de sintomatología ansiosa y depresiva, es un resultado menor al reportado por otras investigaciones en la región, pese a que los instrumentos no permiten la comparación.

Por otra parte, las condiciones materiales de trabajo de los docentes de educación secundaria de Santiago muestran niveles preocupantes de precariedad laboral, sobrecarga horaria, sobre exigencia ergonómica y carencia de materiales e infraestructura. La sobrecarga horaria se expresa básicamente en el porcentaje de horas lectivas en aula, que superan el máximo legal permitido, la alta cantidad de horas extra dedicadas a tareas docentes (equivalentes casi a la tercera parte de la jornada contratada), la mala calidad de la hora de almuerzo y la inexistencia de minutos de descanso durante la jornada laboral. Respecto a la infraestructura observada, la mayor parte de los colegios visitados cuenta con espacios deficientes para profesores (salas inadecuadas, con mobiliario en mal estado y mala distribución del espacio), respecto a las aulas de clases de observaron deficientes condiciones de iluminación y de aislamiento del ruido.

Las condiciones psicosociales de trabajo de los docentes de educación secundaria de Santiago destacan por los elevados niveles de demanda laboral percibidos. Estos resultados de sobredemandas, unidos a los indicadores de jornada laboral, nos muestran que los profesores de educación secundaria de Santiago están sometidos a fuertes procesos de intensificación laboral, que repercuten negativamente en su bienestar psicológico y salud. La percepción de sobredemanda, pese a tener condiciones externas, tiende a ser más baja cuando los docentes se sienten apoyados en su trabajo por parte de la dirección del establecimiento y cuando perciben tener mayores grados de decisión sobre los procesos de trabajo cotidiano.

Es fundamental analizar estos resultado de intensificación laboral docente a la luz de un conjunto de transformaciones en la organización 
del trabajo docente en América Latina, que han implicado nuevas demandas laborales, básicamente por el cambio en los enfoques pedagógicos deseados, la presión por los rendimientos estandarizados, el aumento en las brechas de desigualdad socioeducativa, la incorporación de nuevos sectores populares a la escolarización, la introducción de la lógica gerencial en la escuela, la sobrecarga de trabajo, la falta de apoyo desde instituciones externas a la escuela, la desvalorización social de la profesión y la construcción de nuevos imaginarios desde los que los profesores definen opciones y compromisos (Martínez, 2003; Cornejo, 2007; Robalino, 2005, Andrade, Gonçalves y Melo, 2004).

El modelo explicativo del bienestar docente quedó constituido por siete variables, que logran explicar un $47 \%$ de la varianza del Índice General de Bienestar. A partir de este modelo podemos afirmar que el bienestar y la salud de los docentes de educación secundaria de Santiago se benefician por la posibilidad de otorgarle un propósito moral trascendente y un sentido no instrumental al trabajo educativo y se malogran por el alto nivel de carga de trabajo que perciben los docentes.

El modelo explicativo del bienestar/malestar docente en profesores de educación secundaria de Santiago nos muestra, en toda su dimensión, las paradojas del ser docente en Santiago de Chile. Llama la atención la incorporación de la variable dependencia municipal en el modelo explicativo, como una variable que aporta al bienestar docente; tomando en cuenta que en establecimientos municipales se aprecian peores características de infraestructura, mayores niveles de exigencia ergonómica y mayor nivel de violencia en el entorno social de la escuela. Sin embargo, es en estos colegios donde los docentes trabajan con estudiantes con mayores niveles de vulnerabilidad educativa, lo cual también es un elemento explicativo del bienestar docente. Aunque es preciso indagar más acerca de estos hallazgos, se puede pensar que la construcción de un propósito moral sobre el trabajo guarda relación con el sentido social del mismo, que puede aumentar al trabajar con estudiantes más vulnerables. Por otra parte, en el sector municipal algunas condiciones materiales de trabajo son más favorables, tales como mayor estabilidad laboral, mayores niveles de sindicalización y mayores niveles de remuneración.

Paradójicamente, las exigencias ergonómicas, mayores en el sector municipal, son explicativas del malestar docente, particularmente el estar obligado a forzar la voz permanentemente y trabajar en un ambiente 
Condiciones de trabajo y bienestar/malestar docente en profesores...

ruidoso. Lo mismo se puede decir de la existencia de procesos de selección de estudiantes que explican, en algún porcentaje, las variaciones en el bienestar docente, y que se presentan mayormente en establecimientos particulares subvencionados.

Finalmente, el modelo explicativo del bienestar/malestar docente presentado apoya aspectos centrales de los dos principales modelo en discusión actualmente: el modelo control-demanda y el modelo desajuste esfuerzo-recompensa. A su vez es concordante con la evidencia mundial que plantea la importancia de los propósitos morales en el trabajo docente y los peligros de la intensificación del trabajo educativo (Fullan, 1993; Marchesi, 2007).

Por último, es necesario seguir indagando acerca del sentido de lo público en el trabajo docente, que aparece en este estudio desde las perspectivas de la construcción de sentido en el trabajo, de la vulnerabilidad de los jóvenes y del sistema mismo de educación pública.

Recebido em maio de 2009 e aprovado em julho de 2009.

\section{Nota}

1. Para la construcción del cuestionario de autorreporte se utilizaron, como referentes, los siguientes instrumentos: MBI-ES (Maslach, 2003), Test GHQ-12 (Goldberg), Cuestionario de Calidad del Trabajo para Profesores de Leiden LAKS-Doc (van der Doef y Maes, 1999), Manual para la evaluación de riesgos psicosociales en el trabajo (Método ISTAS 21) (Moncada, Llorens y Kristensen 2000), Cuestionario de condiciones de trabajo SAT-DOC de UNESCO (2005).

\section{Referencias}

ANDRADE, D.; GONÇALVES, G.; MELO, S. Cambios en la organización del trabajo docente: consecuencias para los profesores. Revista Mexicana de Investigación Educativa, México, v. 9, n. 20, p. 183197, enero/marzo 2004.

CODO, W. (Coord.). Educação: carinho e trabalho. Petrópolis: Vozes, 1999.

CORNEJO, R. El trabajo docente en la institución educativa: los procesos de apropiación/enajenación en el trabajo en el contexto de 
las reformas educativas. Revista de Psicología, Santiago, v. 15, n. 2, p. 5-18, 2006.

CORNEJO, R. Salud laboral docente: entre el sufrimiento individual y los sentidos colectivos. Revista Docencia, Santiago de Chile, n. 35, p. 35-54, ago. 2008.

CORNEJO, R.; QUIÑÓNEZ, M. Factores asociados al malestar/ bienestar docente. Revista Electrónica Iberoamericana sobre Calidad, Eficacia y Cambio en Educación, Madrid, v. 5, n. 5e., dic. 2007.

DEJOURS, C. Trabajo y desgate mental: una introducción a la psicopatología del trabajo. Buenos Aires: Humanitas, 1990.

DÍAZ, A.; INCLÁN, C. El docente en las reformas educativas: sujeto o ejecutor de proyectos ajenos. Grupo de trabajo "Educación, trabajo y exclusión social”. CLACSO, 2001.

FULLAN, M. Las fuerzas del cambio: explorando las profundidades de la Reforma Educativa. Madrid: Akal, 2002.

GOLDBERG, D.; WILLIAMS, P. A user's guide to the general health questionnaire. Berkshire: Nfer-Nelson, 1991.

GUGLIELMI, S.; TATROW, K. Occupational stress, burnout, and health in teachers: a methodological and theoretical analysis. Review of Educational Research, Washington, DC, v. 68, n. 1, p. 61-99, 1998.

HARGREAVES, A. Profesorado, cultura y postmodernidad: cambian los tiempos, cambia el profesorado. Madrid: Morata, 1996.

KARASEK, R.; THEOREL, T. Healthy work, stress, productivity and the reconstruction of working life. New York: Basic Books, 1990.

KYRIACOU, C. Teacher stress: directions for future research. Educational Review, Edinburgh, v. 53, n. 1, 2001.

MARTÍNEZ, D. Abriendo el presente de una modernidad inconclusa: treinta años de estudios del trabajo docente. Buenos Aires: Instituto de Investigaciones Pedagógicas; Confederación de Trabajadores de la Educación de la República, 2001. 
Condiciones de trabajo y bienestar/malestar docente en profesores...

MASLACH, C. Job burnout: new directions in research and intervention. Current Directions in Psychological Science, v. 12, n. 5, p. 189-192, 2003.

MENDEL, G. La sociedad no es una familia: del psicoanálisis al sociopsicoanálisis. Argentina: Paidós, 1993.

MONCADA, S.; LLORENS, C.; KRISTENSEN, T.S. Manual para la evaluación de riesgos psicosociales en el trabajo: (método ISTAS 21CoPsoQ). Barcelona: Instituto Sindical de Trabajo, Ambiente y Salud (ISTAS); Paralelo, 2002.

MONTGOMERY, C.; RUPP, A. A meta-analysis for exploring the diverse causes and effects of stress in teachers. Canadian Journal of Education, Toronto, v. 28, n. 3, p. 458-486, 2005.

PARRA, M. Salud mental y trabajo. In: Monografias de Gestión en Psiquiatria y Salud Mental. Santiago de Chile: Universidad de Santiago de Chile, 2001. Disponible en <www.psiquiatriasur.cl >. Obtenido en: mayo 2007.

RASKU, A.; KINNUNEN, U. Job conditions and wellness among finish upper secondary school teachers. Psychology and Health, Chur, v. 18, n. 4, p. 441-456, 2003.

ROBALINO, M. ¿Actor o protagonista?: dilemas y responsabilidades sociales de la profesión docente. Revista PRELAC, Santiago de Chile, n. 1, jul. 2005.

TENTI, E. (Comp.). El oficio de docente: vocación, trabajo y profesión en el siglo XXI. Buenos Aires: IIPE/UnesCo; Siglo Veintiuno, 2007.

UNESCO. Condiciones de trabajo y salud docente: estudios de casos en argentina, Chile, Ecuador, México, Perú y Uruguay. Santiago de Chile: UNESCO-OREALC, 2005.

VAN DER DOEF, M.; MAES, S. The Job-Demand-Control-(Support) model and psychological wellbeing: a review of 20 years of empirical research. Work and Stress, London, n. 13, p. 87-114, 1999.

VERHOEVEN, C. et al. Job conditions and wellness/health outcomes in Dutch secondary school teachers. Psychology and Health, Chur, v. 18, n. 4, p. 473-487, 2003. 